\title{
The fisiopsico-oncology rehabilitation for encapsulation and the asymmetry of the prosthetic implant breast
}

\section{Opinion}

Reconstructive surgery has made it possible that a woman with breast cancer can feel her breasts with an almost equal shape and texture to each other due to new experiences in the field of reconstruction of the breast capsule. The advanced technique of autologous fat transplantation (TGA) allows that, after a mastectomy or quadrantectomy, we can implant the fat of the patient herself. It will be taken from the thighs or abdomen, after being analyzed will be injected on the thoracic region (breast sulcus, above the chest muscle) to recreate the breast capsule where you cannot insert a breast implant, or the implant presenting cutaneous depressions to correct the imperfections after surgery. The treatment will be performed at various stages taking into account the conditions of the patient and calculating the risk of outbreaks of metastasis or recurrence in such a way that the injection of platelets and plasma contained nell'adipe, do not present risks to the patient. The TGA technique has been studied and improved in such a way that the woman undergoes a breast cancer may have not only the perception of the mammary gland but that in the observation and palpation has a self-image more secure. Breast aesthetic appearance with plastic reconstruction may not always be decisive and also depends on the structure which has the pectoral muscle. Each patient will have a physique and a history, it will take into account the type of cancer, previous treatment, the general state of the patient, invasion of tissues and organs and medical therapies that have to do (eg radiotherapy) and based on these factors will be rebuilt the capsule.

After the surgery there will be the disappearance of edema post operative, and it will decide if it is the case de intervene with the TGA technique in such a way that if the patient experiences of the depressions can be corrected with the lipofilling technique. The complications that may arise after the plastic reconstruction are encapsulation and the asymmetry of the breast implant. The encapsulation of the prosthesis the factors that cause this phenomenon may be the fibrous tissue that surrounds the breast, skin, the effects of radiotherapy, previous skin diseases, the elasticity of the fabric and the weight gain. The remainder of the residual scar that with the passage of time imprisoning envelops the capsule in such a way that loses the total or partial mobility by presenting a hard consistency and sore. Another cause of the inflammation of the capsule may be the presence of bacteria that may be present from the same catheter that the patient port in the operating room and that involves total removal of the implant. ${ }^{1}$

The capsule may be too rigid, higher than the other, larger or smaller. When it presents a factor of this type you must be surgically intervene with the capsulectomy to proceed with removal and replacement of the breast prosthesis implant. The mammary gland has as structures the nerves and lymph vessels which has the name in addition to the milk ducts and the furriers muscles, it has adipose tissue that changes with the passage of weight aging or for the variation of elasticity and contributing to ptosis (sagging Clearly if you were to make a removed
Volume 6 Issue 4 - 2016

\author{
Stella Maris Glowinski \\ Riabilitazione Oncologica, Italy
}

Correspondence: Stella Maris Glowinski,Via nomentana 455 00162 Roma, Italy, Tel 333-5422456,

Email stella.glowinski@gmail.com

Received: November 25, 2016 | Published: December 16, 2016

all the breast structure remaining a Following the installation with the capsule and lipofilling. The patient and very important, and you fat the negative effects on the asymmetry between the two an increase in weight, will also increase the weight in Cooper's ligaments, a component of both for the time patient losing breast). Mastectomy will furrow where he will Body Weight lose weight can lead breasts, if the patient has Breast due to component of fatty tissue surrounding the breast.

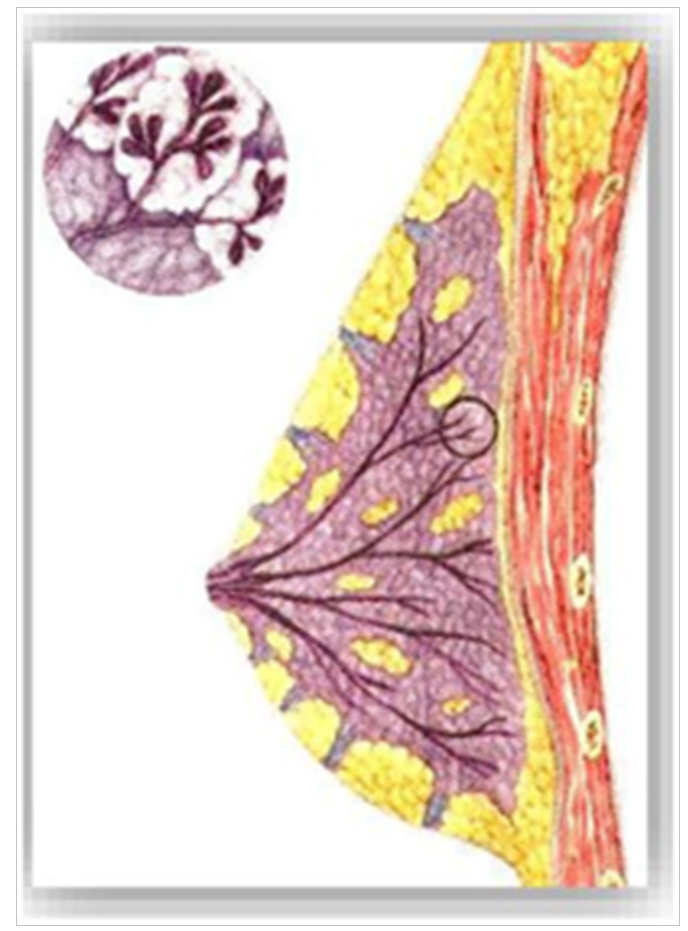

Figure I Constitution of the mammary gland. 
Early physical therapy can be an effective support as soon as the patient is able to follow a physical treatment and involves manual massage, linfotaping and isometrics and posture correction. Lymphatic drainage and linfotaping after reconstruction not only favor the elimination of edema local breast but also facilitate the movement of freeing capsule of fibrotic residuals that it may present. The compression exerted on the system when fluids are not removed can also create stillness plant also causing an asymmetry in the reconstructed breast. In this phase, as well as follow a therapy with circular movements, we follow the isometrics for the pectoral muscle and the group of the scapular humeral muscles with breathing trying to push the diaphragm towards the abdomen in such a way that recovers elasticity and functionality to support the capsule. Moreover, the involvement of the patient is very important, many of them are in the phase of denial for breast loss and sometimes fail to touch the wound so physiotherapy area is also trying to involve the patient to follow a self-massage in a circular fashion with two hands the therapist will teach the patient as well as to do gymnastics at home promote the progress of the therapy. Educate and inform a woman with breast cancer is not an easy task, but they spend many hours in rehabilitation and physical therapist is privileged to interact with them by listening and giving the best advice for the future physical and mental wellbeing including education food. It is not part of the role of physical therapist giving a diet, but you explain the reasons for the increase or decrease in body weight can affect the asymmetry or encapsulation of the breast implant. The cancer patient undergoes physical changes continuously from the time of surgery, with hormone treatment until the end of care established by the medical protocol. There will be joint pain, muscle and swelling of the hands and feet as the trunk and abdomen with weight changes and all these factors increase the risk that the patient is in one is the least of his thoughts was fragile and emotional power. Functional nutrition is very important, taking the food changes and change every cell of our bodies, this means that at every meal we can modify our body going towards health or towards the disease and everything depends on what mangiamo. ${ }^{2}$ Fat which is deposited in the abdomen it is also deposited in the circumference of the breast or of the remaining groove after the removal of the breast.
If your body fat also increases there will an increase in the ipsilateral and contralateral breast, the difference lies in the healthy breast has an autonomous mobility instead Breast with no implants, both for its composition and for the points that secure it to the wall thoracic and muscle. This fat can cover the dish and wrap depriving it of the movement that has within the breast cavity. When this happens inflammatory process must remove the implant and dig further into the adipose tissue to remove the fibrous plaque that impedes movement and free it, this involves an additional space that can determine an additional asymmetry between the two breasts. The importance of education fisiopsico-cancer after a breast cancer has a much more expanded vision that allows through the ability of the operator, inform and follow the patient in a comprehensive manner, both a sanitary aspect in terms of aesthetics for the well being of the person. The auto massaging technical and Food rehabilitation can avoid many discounts for women living this disease and will depend very much on the sensitivity of the health professional who will be responsible to follow treatment do get the concept of self-esteem and exploitation through the care physiotherapy to all women who have lost such an important part of himself. ${ }^{3}$

\section{Acknowledgments}

None.

\section{Conflicts of interest}

Author declares there are no conflicts of interest.

\section{References}

1. Modena S. Breast Treaty. Piccin (Padua): Italy; 2006. 647 p.

2. Sara Farnetti. Internist nutritionist for diet B Functional. Rome, Italy; 2016.

3. Dominici L. Constitution of the mammary gland, plastic surgery breast Piccin Nuova Libraria: Padova, Italy; 1989. 